'BS Karagol, 'A Zenciroglu, 'N Okumus, ${ }^{2}$ RA Polin. 'Dr Sami Ulus Maternity, Childrens Education and Research Hospital, Division of Neonatology, Ankara, Turkey; ${ }^{2}$ Columbia University College of Physicians and Surgeons, NY Presbyterian Morgan Stanley Children's Hospital, Division of Neonatology, New York, NY, USA

Objective To evaluate the effect of slow versus rapid rates of advancement of enteral feed volumes upon the clinical outcomes in preterm infants with 750-1250g birth weight.

Study Design A total of 92 stable neonates with the birth weight $750-1250 \mathrm{~g}$ and gestational age less than 32 weeks were randomly allocated to enteral feeding advancement of $20 \mathrm{ml} / \mathrm{kg} /$ day $(\mathrm{n}=46)$ or $30 \mathrm{ml} / \mathrm{kg} /$ day $(\mathrm{n}=46)$. The primary outcome was days to reach full enteral feeding defined as $180 \mathrm{ml} / \mathrm{kg} /$ day. Secondary outcomes included rates of necrotizing enterocolitis and culture-proven sepsis, days of total parenteral nurition, length of hospital stay and growth end points.

Result Neonates in the rapid feeding advancement group achieved full enteral volume of feedings earlier than the slower advancement group. They received significantly fewer days of parenteral nutrition, exhibited a shorter time to regain birth weight and shorter duration of hospital stay. The incidence of NEC and the number of episodes of feeding intolerance were not significantly different between the groups while the incidence of culture-proven late onset sepsis was significantly less in infants receiving a rapid feeding advancement. Excluding infants who were small for gestational age at birth, the incidence of extrauterine growth restriction was significantly reduced in the rapid advancement group at 28 days and at hospital discharge.

Conclusion Rapid enteral feeding advancements in 750-1250g birth weight infants reduces the time to reach full enteral feeding and the use of TPN administration. Rapid advancement enteral feed also decreases extrauterine growth restriction with improved shortterm outcomes for these high-risk infants.

\section{A RANDOMISED TRIAL COMPARING FISH OIL (SMOFLIPID) VS. OLIVE OIL LIPID EMULSION (CLINOLEIC) IN PRETERM $(<30$ WEEKS) NEONATES}

doi:10.1136/archdischild-2012-302724.0103

${ }^{1,2} \mathrm{G}$ Deshpande, ${ }^{3} \mathrm{M}$ Deshmukh, $3,4 \mathrm{~K}$ Simmer, ${ }^{5} \mathrm{~T}$ Mori, ${ }^{5} \mathrm{~K}$ Croft, ${ }^{6} \mathrm{~J}$ Kristensen. 'Neonatology, Nepean Hospital Sydney; ${ }^{2}$ Nepean Clinical School, University of Sydney, Kingswood; ${ }^{3}$ Neonatology, King Edward Memorial Hospital, Perth, NSW; ${ }^{4}$ Centre for Neonatal Research and Education, School of Womens and Infants Health, University of Western Australia, Perth, WA; ${ }^{5}$ School of Medicine and Pharmacology, University of Western Australia; ${ }^{6}$ Pharmacy, King Edward Memorial Hospital for Women, Perth, NSW, Australia

Background Fat emulsions used in Australia for $\mathrm{PN}$ in preterm neonates have been based on either soybean oil (SO) or olive oil (OO). 00 based lipid Clinoleic has high ratio of $n-6$ to $n-3$ fatty acids (9:1) this may not be ideal for LC-PUFA supply. Newly available SMOFlipid has appropriate ratio n-6 to n-3 fatty acids (2.5:1). SMOFlipid also contains $\mathrm{OO}(25 \%)$, coconut oil (30\%) and SO $(30 \%)$. Better lipid clearance, reducing the risk of liver toxicity, reduced oxidative stress, lower immune-activity and antiinflammatory effects are other potential advantages of SMOFlipid. Method Preterm neonates (23-30 weeks) were randomised to receive Clinoleic or SMOFlipid emulsion for 7days using a standard protocol. Investigators and outcome assessors were masked to allocation. Plasma $\mathrm{F}_{2}$-Isoprostanes (lipid peroxidation marker), $\mathrm{RBC}$ fatty acids, vitamin-E were measured before and after the study. Blood culture positive sepsis and growth was monitored for safety.

Results 30/34 participants (Clinoleic-15, SMOFlipid-15) completed the study. Both emulsions were well tolerated without any adverse events. $\mathrm{F}_{2}$-isoprostane levels were reduced in SMOFlipid group as compared to baseline. Eicosapentanoic acid (EPA) and
vitamin-E levels were significantly increased in SMOFlipid group. Oleic and Linoleic acid levels were increased in both groups. No significant differences were noted in post study Docosahexaenoic acid (DHA) levels in both groups despite higher levels of DHA in SMOFlipid.

Conclusions SMOFlipid was safe, well tolerated and also showed beneficial effect in terms of reduction of oxidative stress by reducing lipid peroxidation levels in high risk preterm neonates.

\section{CEREBRAL AUTOREGULATION IN THE NEWBORN}

doi:10.1136/archdischild-2012-302724.0104

T Austin. Neonatal Intensive Care, Cambridge University Hospitals NHS Foundation Trust, Cambridge, UK

Vascular pressure reactivity is the ability of vascular smooth muscle to respond to changes in transmural pressure. In the cerebral circulation this reactivity - or autoregulation - limits cerebral blood flow variation over a range of cerebral perfusion pressures ensuring adequate perfusion and oxygenation to the brain.

In adults cerebrovascular pressure reactivity can be determined by observing the response of intracranial pressure (ICP) to changes in mean arterial blood pressure. Non-invasive techniques such as transcranial Doppler ultrasound and near-infrared spectroscopy have been validated against ICP measurements, which have enabled continuous assessment of cerebral autoregulation to be investigated in newborn infants.

A number of different techniques have been described, including static and dynamic measurements and analysis in the time and frequency domain, yet despite many years of research the characteristics of cerebral autoregulation in the newborn are still not clear.

Both the presence and limits of autoregulation has been much debated although there is increasing evidence that autoregulation, while present in healthy infants, is impaired in sick term and preterm neonates and that this impairment may be a predictor of poor outcome.

In clinical practice there is a reliance on blood pressure measurements alone to make informed clinical decisions, which ignores the complex circulatory control mechanisms that exist to optimize oxygen delivery to the brain. The ability to obtain continuous quantitative information on cerebral autoregulation at the cotsie would represent a significant advance in the management of these patients.

\section{NON-INVASIVE MEASUREMENTS OF HEMODYNAMIC TRANSITION AT BIRTH}

doi:10.1136/archdischild-2012-302724.0105

${ }^{1} \mathrm{JJ}$ van Vonderen, ${ }^{2} \mathrm{AA}$ Roest, ${ }^{3} \mathrm{ML}$ Siew, ${ }^{1} \mathrm{FJ}$ Walther, ${ }^{3} \mathrm{SB}$ Hooper, ${ }^{1} \mathrm{AB}$ te Pas. ${ }^{1}$ Division of Neonatology, Department of Pediatrics; '2Department of Pediatric Cardiology, Leiden University Medical Center, Leiden, The Netherlands; ${ }^{3}$ itchie Centre, Monash University, Melbourne, VIC, Australia

Objective To investigate the hemodynamic changes during transition at birth obtaining non-invasive physiological data in healthy term infants.

Methods In 18 newborns (GA 39 weeks (38-39)) born by a caesarian section; arterial oxygen saturation $\left(\mathrm{SaO}_{2}\right)$ (preductally), heart rate $(\mathrm{HR})$ and non-invasive blood pressure $(\mathrm{BP})$ were measured and echocardiography using $\mathrm{M}$-mode and Doppler flow was performed at 2, 5 and 10 minutes after birth

Results Oxygen saturation and HR were within recommended target ranges. Mean BP did not change between measurement intervals $(55 \mathrm{~mm} \mathrm{Hg}$ at $2 \mathrm{~min}, 54 \mathrm{~mm} \mathrm{Hg}$ at $5 \mathrm{~min}$. and $54 \mathrm{~mm} \mathrm{Hg}$ at $10 \mathrm{~min}$ ) and was similar as BP measured at day 1 . Left ventricle output (LVO) significantly increased between $2 \mathrm{~min}$ and $5 \mathrm{~min}$ 
(120.3 $\mathrm{mL} / \mathrm{kg} / \mathrm{min}$ (35.1) vs. $167.4 \mathrm{~mL} / \mathrm{kg} / \mathrm{min}$ (67.3); $\mathrm{p}$ value $<0.001)$ but remained stable by $10 \mathrm{~min}(189.2 \mathrm{~mL} / \mathrm{kg} / \mathrm{min}(26.2)$; $\mathrm{ns})$. The increase in LVO was significantly correlated to an increase in HR and left ventricle end diastolic diameter $(14.3 \mathrm{~mm}$ at $2 \mathrm{~min}$, $16.0 \mathrm{~mm}$ at $5 \mathrm{~min}$ and $16.6 \mathrm{~mm}$ at $10 \mathrm{~min} ; \mathrm{p}<0.001)$. We observed a bidirectional ductal flow in the first minutes, but large inspirations following a cry overruled the bidirectional ductal flow causing a large left to right shunting.

Conclusion At birth, the most significant hemodynamic change occurred in the first 5 minutes whereby an increase in preload and heart rate increased LVO. Inspirations had a major influence on ductal flow, causing large left to right shunting. Blood pressure at birth remained stable.

\section{EARLY POSTNATAL CHANGES IN SPLANCHNIC CIRCULATION IN HEALTHY TERM NEWBORNS}

doi:10.1136/archdischild-2012-302724.0106

${ }^{1} \mathrm{M}$ Zibolen, 'E Paulusova, ${ }^{2} \mathrm{~J}$ Zibolenova, 'L Kocvarova, ' $\mathrm{K}$ Matasova. ${ }^{1} \mathrm{Clinic}$ of Neonatology; ${ }^{2}$ Dept. of Public Health, Jessenius Medical Faculty Comenius University, Martin, Slovak Republic

Objective To characterize hemodynamic changes in celiac artery (CA) and superior mesenteric artery (SMA) in healthy term newborns during the first six hours after birth by means of Doppler ultrasonography.

Materials and methods The study included 30 healthy term newborns. The ultrasound examinations using Aloka $\alpha-10$ were performed at the age of 2.4 and 6 hours after birth. The statistical analysis of Doppler variables was performed using the non - parametric Friedman test with all pairwise multiple comparisons. Presented results are shown as median (interquartile range).

Results A reversed blood flow in SMA, documented by negative values of end diastolic velocity (EDV) [-10.90 cm.s-1 (-13.21-0.00)], changed to positive during the first six hours after birth $(7.08 \mathrm{~cm} . \mathrm{s}-1$ (6.29-13.48), $p<0.001)$. In CA, EDV increased in this period, but negative values were not recorded [11. 83 cm.s-1 (9.34-13.86) and 18.03 cm.s-1 (14.20-27.57) ].

Conclusion Dramatic changes occur in splanchnic circulation during the first six hours after birth. In superior mesenteric artery, the negative diastolic blood flow turns to positive as a part of circulatory transition in the presence of a ductal steal.

Acknowledgment This work was supported by project "Center of Excellence of Perinatology Research (CEPV II)", ITMS code: 26220120036, which is co-financed by EU sources.

\section{SURVIVAL OF VERY PRETERM CHILDREN BORN IN 2011: FIRST RESULTS OF THE EPIPAGE 2 COHORT STUDY}

doi:10.1136/archdischild-2012-302724.0107

1.2PY Ancel. 'INSERM Unit 953, Pierre-et-Marie-Curie University; ${ }^{2}$ INSERM Unit 953, Hopital TENON - APHP, Paris, France

\footnotetext{
Aims The number of neonates born preterm is increasing and children born at low gestational ages face a range of risks. We present the first results of the EPIPAGE 2 study, a national cohort of very and moderately preterm children in France.

Methods EPIPAGE 2 is a prospective population-based cohort study conducted in 25 regions of France in 2011 including extremely (22-26 weeks, 8-month recruitment), very (27-31 weeks, 6-month recruitment) and moderately preterm (32-34 weeks, 1-month recruitment) children. It aims to examine short and long term outcomes (survival, health and development) of these children and their determinants. Data on pregnancy, delivery, and neonatal events were extracted from the obstetric and neonatal records.
}

Results A total of 5565 births were included in the EPIPAGE 2 study: 1752 were born extremely preterm (898 (51\%) stillbirths and 854 (49\%) live births), 2728 very preterm (298 (11\%) stillbirths and 2430 (89\%) live births), and 1085 moderately preterm (28 (2.5\%) stillbirths and 1057 (97.5\%) live births). The survival rates for extremely preterm babies were $23 \%$ of all births and $48 \%$ of live births. The corresponding survival rates were respectively $83 \%$ and $93 \%$ at $27-31$ weeks and $96 \%$ and $99 \%$ at 32-34 weeks. In 1997 (EPIPAGE 1 study), the survival rates were $20 \%$ of all births and $46 \%$ of live births at $22-26$ weeks and $76 \%$ of all births and $88 \%$ of live births at 27-31 weeks.

Conclusion Survival has improved among very preterm children, but has not changed at extremely low gestational ages.

\section{ALTERED FRONTO-TEMPORAL NEUROANATOMY IN VERY PRETERM BORN ADULTS DURING COMPLETION OF A VERBAL FLUENCY TASK}

doi:10.1136/archdischild-2012-302724.0108

${ }^{1,2} \mathrm{~N}$ Garcia Casares, 'S Froudist Walsh, 'MP Allin, 'M Walshe, 'RM Murray, 'C Nosarti. 'Department of Psychosis Studies, Institute of Psychiatry, King's College London, London, UK; ' ${ }^{2}$ Centro de Investigaciones Medico-Sanitarias (CIMES), C/Marques de Beccaria 3, Malaga, Spain

Background and aims Several studies have described poorer cognitive performance in various aspects of language such as comprehension, naming, expressive language and verbal fluency in individuals who were born very preterm compared to controls. Neuroimaging investigations have also described structural and functional changes that could underlie such language deficits. In this pilot study we used functional MRI to explore whether alterations in the neuroanatomy of phonological verbal fluency persisted into adulthood following very preterm birth.

Methods Twenty-two young adults (26-28 years old, females=11, males=11) were examined. Eleven individuals were born very preterm (gestational age: $<33$ weeks) and 11 were full-term born controls. All participants underwent functional MRI using a 3T scanner while completing a phonological verbal fluency task. All data pre- and postprocessing was performed using FSL (www.fmrib.ox.ac.uk/fsl).

Results During successful verbal fluency trials, very pretermborn individuals showed significantly decreased blood-oxygenlevel-dependent signal compared to controls in several brain areas including the orbitofrontal/medial frontal cortex bilaterally $(x=8 \mathrm{~mm}, y=52 \mathrm{~mm}, z=-28 \mathrm{~mm})$ and the left fusiform/parahippocampal gyrus $(x=-34 \mathrm{~mm}, y=-30 \mathrm{~mm}, \mathrm{z}=-24 \mathrm{~mm}), \mathrm{p}<0.05$ family wise-error corrected (FWE). On-line performance did not significantly differ between the groups.

Conclusions Previous studies with functional MRI in younger samples have shown neuroanatomical alterations (increased and decreased activation) in fronto-temporal and fronto-striatal networks in very preterm born individuals when performing different cognitive tasks. Our results suggest that neuroanatomical alterations during language processing persist into adult life following very pre-term birth, and specifically involve fronto-temporal networks, with important implications for a wide range of high order cognitive functions.

\section{NEONATAL CEREBRAL OXYGENATION IS ASSOCIATED WITH NEURODEVELOPMENTAL OUTCOME OF PRETERM INFANTS AT 2 TO 3 YEARS OF AGE}

doi:10.1136/archdischild-2012-302724.0109

'EA Verhagen, 'KNJA Van Braeckel, 'CN van der Veere, ${ }^{2} \mathrm{H}$ Groen, ${ }^{1} \mathrm{PH}$ Dijk, ${ }^{1} \mathrm{CV}$ Hulzebos, 'AF Bos. 'Division of Neonatology, Beatrix Children's Hospital; ' 2 Department of Epidemiology, University of Groningen, University Medical Center Groningen, Groningen, The Netherlands 